\title{
Numerical Investigation on Aerodynamic Force of Streamlined Box Girder with Uniform Air Suction
}

\author{
Tang Ke ${ }^{1, *}$, Shi Cong ${ }^{1}$, Zhou ZhiYong ${ }^{2}$, Yang HanXiang ${ }^{3}$ and Sungur P.T ${ }^{4}$ \\ ${ }^{I}$ Southwest Jiaotong University, Sichuan, 610202, China \\ ${ }^{2}$ Chongqing rail transit (group) Co.,Ltd, 400000, China \\ ${ }^{3}$ Sichuan Chuanjiao Road\&Bridge Co.,Ltd, 618300, China \\ ${ }^{4}$ Arizona State University, AZ 85246, United States
}

Received 4 January 2014; Accepted 30 June 2014

\begin{abstract}
In the present study, the flow around a streamlined box girder with uniform air suction has been investigated numerically. Two-dimensional incompressible unsteady Reynolds averaged Navier-Stokes (URANS) equations are solved in conjunction with the SST $k-\omega$ turbulence model in simulations. Taking the Great Belt Bridge girder as an example, cases of different suction positions on the girder section were discussed. The effect of the suction ratio and the angle of attack (AOA) of wind also were investigated. The result showed that the aerodynamic drag force was influenced by the uniform suction through either upper surface or lower surface of the box girder. The larger the suction ratio was, the more the drag-reducing could be. The suction position and AOA had a comprehensive effect on the drag force. The vortex shedding frequency was also affected by air suction. For the aerodynamic lift force and moment, air suction showed no obvious influence. If necessary, using a combined suction scheme to reduce the aerodynamic drag force or to control the flow wake would be more efficient in engineering design.
\end{abstract}

Keywords: Streamlined Box Girder, Uniform Air Suction, URANS, Suction Ratio, Angle of Attack

\section{Introduction}

The bridge, usually counted as the most important key transport infrastructure, has experienced a rapid development period in China. More and more world-class long span bridges have been constructed during the past 30 years. A longer span bridge is more susceptible to strong wind because of its weaker stiffness and lower damping. The aerodynamic forces and wind-induced vibrations become the governing factors in long span bridge design. Researchers before have done much work to improve the bridge wind resistance performance.

Nowadays, it is well understood that methods of improving the geometrical configuration of bridge deck cross section make the bridge have a better performance in strong wind. This kind of method works by changing the air boundary layer around the bridge deck passively and requires no external energy.

By contrast, the study of active method which requires energy input is still at its beginning. Flow suction, a typical active method used to control the characteristics of boundary layers, is a focused issues in the fields of computational and experimental fluid dynamics [1-2]. A few literatures have been published about the effects of air suction and blowing

*E-mail address:: monkeytk@163.com

ISSN: 1791-2377 @ 2014 Kavala Institute of Technology. All rights reserved. on bluff body.

Ling investigated the effects of suction position and strength on the vortex around a circular cylinder. The suction on shoulder of cylinder caused the lift force to reduce greatly [3].

Fransson conducted experiment on the flow around a porous circular cylinder with air suction. Results showed that the drag force coefficient drastically decreased linearly with the suction rate. It was found that the Strouhal number increased with suction [4].

Luo used the air suction technique to study the vortex shedding from a circular cylinder [5]. By inducing parallel vortex shedding, the span wise averaged coefficient of drag became marginally higher, while the span wise averaged Strouhal number remained practically unchanged.

Chen used a suction flow method to mitigate vortexinduced vibration of a circular cylinder. When the ratio of the suction velocity to the oncoming flow was less, the suction flow control method was found to behave better for VIV suppression [6].

Shtendel applied an active flow control system by suction and oscillatory blowing to research the drag reduction and wake stabilization of a circular cylinder. The system seemed efficient and robust [7].

Dong studied an effective technique for to suppress the vortex-induced vibration of a circular cylinder numerically. The result revealed that small amounts of combined 
Tang Ke, Shi Cong, Zhou ZhiYong, Yang HanXiang and Sungur P.T

/Journal of Engineering Science and Technology Review 7 (2) (2014) 190 - 196

windward suction and leeward blowing led to suppression of the fluctuating lift force [8].

Cuhadaroglu and Turhal studied the effects of suction and blowing on surfaces of square cylinders by experiment and numerical simulation. Some similar conclusions about the aerodynamic drag force and Strouhal number were also obtained [9-12].

In summary, our review shows that the method of flow suction on circular cylinder and square cylinder has been investigated to a certain extent. However, for bridge girder, the research is still quite limited. Therefore, the main objective of this work is to study the effect of air suction on streamlined box girder section in crosswind. The paper is organized as follows. Section 2 gives a presentation on numerical method, boundary conditions and governing equations. Section 3 is devoted to description of computed cases and the result. Some conclusions are drawn in the last section.

\section{Computational Set-up}

The mesh generator and flow solver used here are the commercial codes ICEM-CFD and Fluent. The velocitypressure is based on a PISO (pressure implicit with splitting of operators) segregated algorithm. For all quantities, spatial and temporal discretizations are performed with 2nd-order schemes. For all simulations, the time step $\Delta t$ is equal to $0.001 \mathrm{~s}$, i.e., about 50 time-step per Strouhal period due to Von Karman vortex shedding.

\subsection{Governing Equations}

Under Boussinesq's assumption, the 2D incompressible unsteady Reynolds averaged Navier-Stokes (URANS) equations are given as follows:

$$
\begin{aligned}
& \nabla \cdot U=0 \\
& \frac{\partial U}{\partial t}+U \cdot \nabla U=-\frac{1}{\rho} \nabla p+\left(v+v_{t}\right) \nabla^{2} U
\end{aligned}
$$

Where $U$ is the mean velocity vector; $v_{t}$ is the turbulent kinematic eddy-viscosity. In this paper, the eddy-viscosity is evaluated by the shear stress transport (SST) $k-\omega$ turbulence model.

The SST $k-\omega$ model proposed by Menter in [13] comprises two transport equations:

$$
\begin{aligned}
& \rho \frac{\partial k}{\partial t}+\rho U_{j} \frac{\partial k}{\partial x_{j}}=\tau_{i j} \frac{\partial U_{i}}{\partial x_{j}}-\beta^{*} \rho k \omega+\frac{\partial}{\partial x_{j}}\left[\left(\mu+\sigma^{*} \mu_{T}\right) \frac{\partial k}{\partial x_{j}}\right] \\
& \rho \frac{\partial \omega}{\partial t}+\rho U_{j} \frac{\partial \omega}{\partial x_{j}}= \\
& \alpha \frac{\omega}{k} \tau_{i j} \frac{\partial U_{i}}{\partial x_{j}}-\beta \rho \omega^{2}+\frac{\partial}{\partial x_{j}}\left[\left(\mu+\sigma \mu_{T}\right) \frac{\partial k}{\partial x_{j}}\right]+\sigma_{d} \frac{\rho}{\omega} \frac{\partial k}{\partial x_{j}} \frac{\partial \omega}{\partial x_{j}}
\end{aligned}
$$

Where the model closure coefficients are

$\alpha=5 / 9, \beta=3 / 40, \beta^{*}=9 / 100, \sigma=1 / 2$,

$$
\sigma^{*}=1 / 2
$$

\subsection{Boundary Conditions and Mesh}

The cross section of Great Belt Bridge (GBB), which is as famous as the first streamlined box girder used for long span bridge in the world, is selected in this investigation. The fullscale section shape and size marking of GBB are illustrated in Fig. 1. In simulations, the section is scaled by a 1:50 ratio to build numerical model with the purpose of cutting down the calculation cost.

As shown in Fig.2, the simulated bridge section is located in the middle and front of the calculation domain. To minimize the obstruction of boundaries on flow pattern around the girder section, the lengths of upstream distance and downstream distance are $5 \mathrm{~B}$ and $20 \mathrm{~B}$, in which $\mathrm{B}$ stands for the width of the girder. These sizes are chosen, which are based on plenty of pilot calculations. The width of the computational domain is assigned $60 \mathrm{H}$, in which, $\mathrm{H}$ stands for the girder height. Thus the blockage ratio would be less than $2 \%$. The boundary conditions are designated as follows:

The symmetry condition in the bottom and top boundaries: $u_{y}=0, \partial u_{y} / \partial y=0$;

Boundary conditions on the suction faces of the girder: $u_{y}=V_{s}, \partial u_{y} / \partial x=0$, where $V_{s}$ is the suction velocity;

Boundary conditions on the no suction walls of the girder: $u=0$

Uniform velocity at inlet: $u_{x}=U_{\infty}, \partial u_{x} / \partial y=0$

Uniform static pressure at outlet: $\partial p / \partial x=0$.

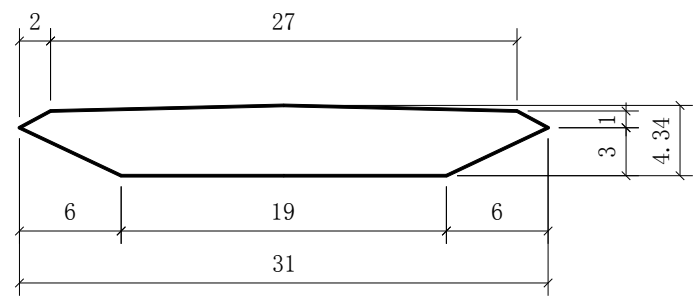

Fig.1 Cross Section of Great Belt Bridge Girder with Lengths in meter.

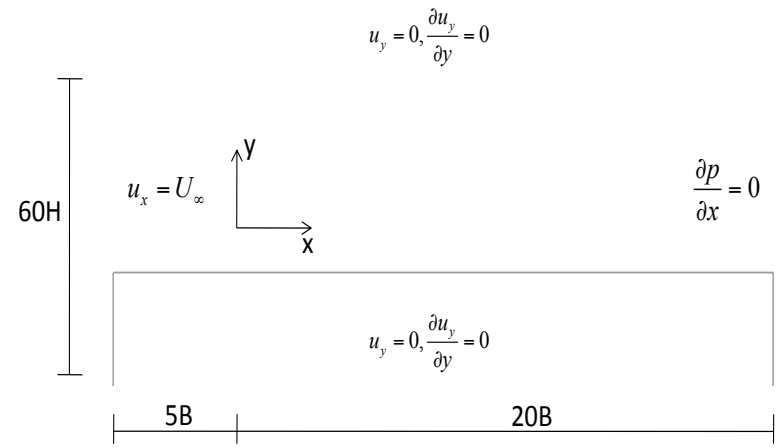

Fig.2 Computational Domain, Boundary Conditions

A non-uniform grid is generated around the girder by using a hybrid grid scheme (see Fig.3). The surrounding region of the computational domain is mapped with structure grid. And nearby the girder is unstructured quadrilateral grid with quite small size in order to satisfy the turbulence model requirement for the first cell thickness. A size function is used to control the gradual change of grid size as long as the distance between the cell positions to the girder surface. The 
Tang Ke, Shi Cong, Zhou ZhiYong, Yang HanXiang and Sungur P.T

/Journal of Engineering Science and Technology Review 7 (2) (2014) 190 - 196

smallest grid size is nearly $0.5 \mathrm{~mm}$. Accordingly, the largest the whole section. The cell number is 294781 in all. nondimensional distance is $y+\approx 5$ on the girder surface over

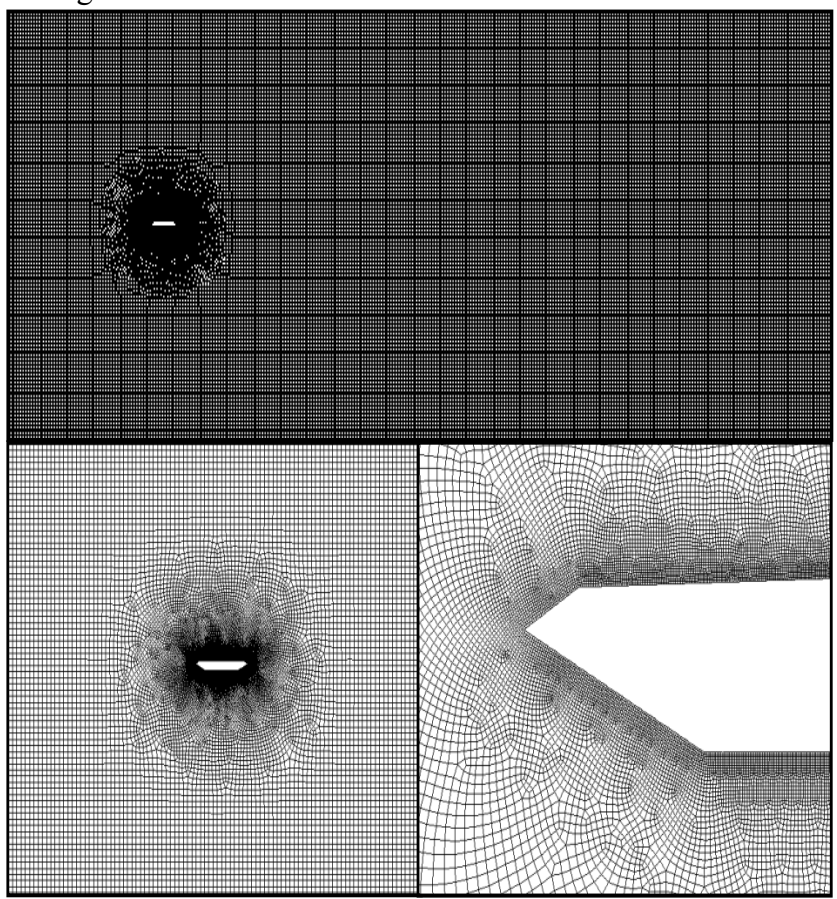

Fig.3 Grid Structure and Details

\subsection{Parameters}

Some basic parameters, including drag coefficient $C_{D}$, lift coefficient $C_{L}$, moment coefficient $C_{M}$, pressure coefficient $C_{P}$, suction ratio $\Gamma$, and Strouhal number $S t$ are defined as following

$$
\begin{aligned}
& C_{D}=\frac{F_{D}}{0.5 \rho U_{\infty}^{2} H} \\
& C_{L}=\frac{F_{L}}{0.5 \rho U_{\infty}^{2} B} \\
& C_{M}=\frac{M}{0.5 \rho U_{\infty}^{2} B^{2}} \\
& C_{P}=\frac{P-P_{\infty}}{0.5 \rho U_{\infty}^{2}} \\
& \Gamma=\frac{V_{s}}{U_{\infty}} \\
& S t=\frac{f_{s} H}{U_{\infty}}
\end{aligned}
$$

Where $F_{D}$ and $F_{L}$ are the force components in alongwind and cross-wind direction respectively; $\rho$ is the air density, $U_{\infty}$ and $P_{\infty}$ are characteristic velocity and pressure of the problem; $H$ is the girder height; $V_{S}$ is the velocity of air suction; $f_{s}$ is the dominate vortex shedding frequency. In this paper, the characteristic Reynolds number $\left(\operatorname{Re}=U_{\infty} H / v\right)$ is equal to 59422 .

\subsection{Suction faces chosen}

The suction position is one of the key issues to control the flow pass by the girder. First of all, the flow around the no suction girder section in cross wind is investigated numerically. Fig. 4 depicts the instantaneous streamlines around the chosen bridge girder. The flow separates at the beginning, and after a short distance, it reattaches to the lower surface of the girder.

As shown in Fig.5a, the mean pressure is negative in a large part of the girder faces except the front area. The largest magnitude of negative pressure occurs at Point 2 for upper surface and Point 6 for lower surface both of which are the first burble point. Two separation bubbles are identified by contours of mean pressure coefficient in flow field seen in Fig.5b.

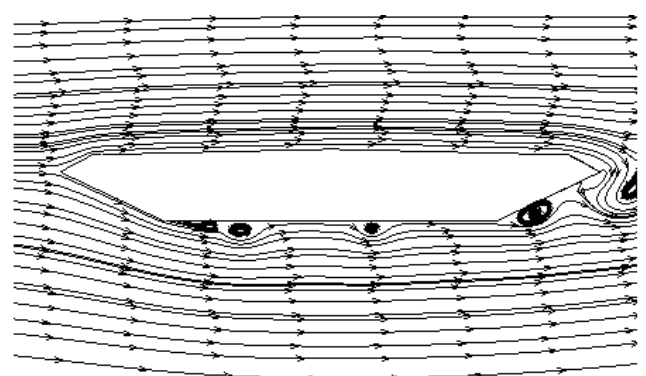

Fig.4 Instantaneous Streamlines Around the Bridge Girder

Based on' the flow mechanism of suction control method, suction faces are fixed close to burble points in this investigation as drawn in Fig.6. Considering the actual achievement in engineering, the area ratio of suction face to girder face $W / B$ is set to $2 \%$. The suction velocity vector $V_{s}$ is perpendicular to the suction face with a positive symbol when the direction is pointing to the girder. 
Tang Ke, Shi Cong, Zhou ZhiYong, Yang HanXiang and Sungur P.T

/Journal of Engineering Science and Technology Review 7 (2) (2014) 190 - 196

As listed in Table 1, applying suction through upper face and lower face separately are labeled as case I and case II . For each case, seven angles of attack of wind to girder section is taken into considered. Effect of different suction ratio is also studied.

(a)
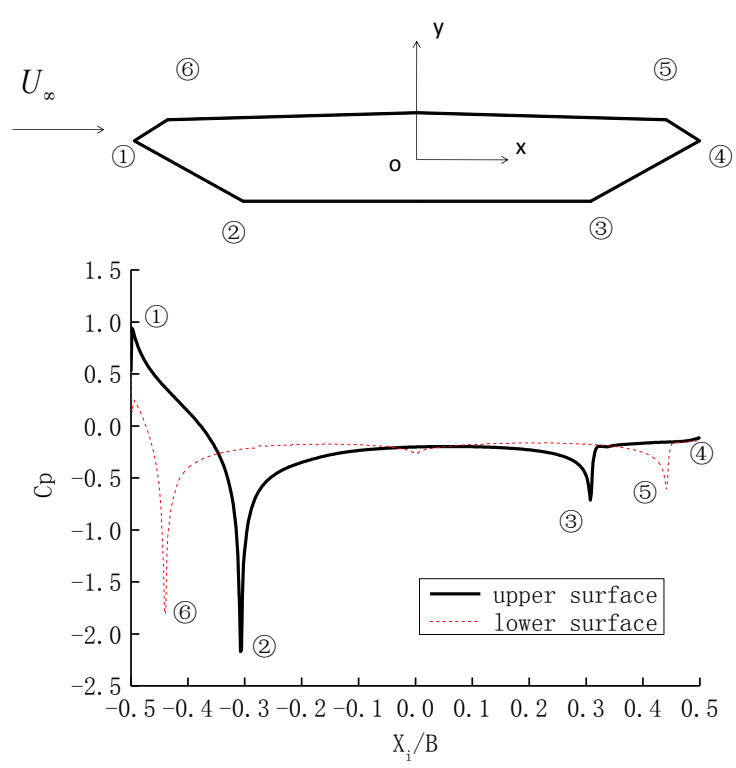

(b)

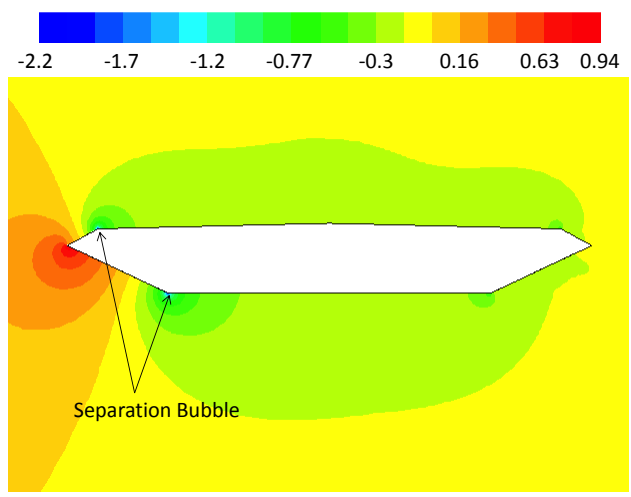

Fig.5 Mean Pressure Coefficient $C_{p}$ on Girder Faces(a) and Distribution in Flow Field(b)

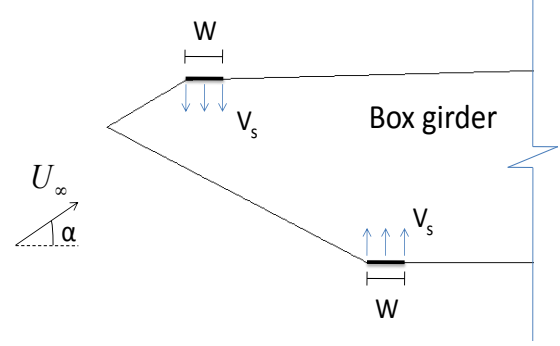

Fig.6 Suction Position on Box Girder

Table 1 Cases Depiction

\begin{tabular}{cccc}
\hline Case Suction position & AOA $\alpha(\mathrm{deg})$ & Suction ratio $\Gamma$ \\
\hline I & upper surface & $-6 \sim+6, \triangle \alpha=2$ & $0 \sim 0.4, \triangle \Gamma=0.1$ \\
II $\quad$ lower surface & $-6 \sim+6, \triangle \alpha=2$ & $0 \sim 0.4, \triangle \Gamma=0.1$ \\
\hline
\end{tabular}

\section{Results and Discussion}

\subsection{Suction on Upper Surface (Case I)}

In this case, suction parameter between $\Gamma=0$ and $\Gamma=0.4$ is employed on the upper surface of girder. When AOA $\alpha=0^{\circ}$, the pressure coefficient distribution around the girder is depicted in Fig.7. Applying suction through the upper surface provides approximately similar pressure distribution on the girder lower surface (Fig.7a). This indicates that the flow under girder cannot be influenced by air suction on upper surface. For the upper surface of girder (Fig.7b), the flow separation is somehow controlled by the air suction ratio. When the suction energy is stronger, for instance $\Gamma=0.4$, the magnitude of negative pressure on upper surface is less.

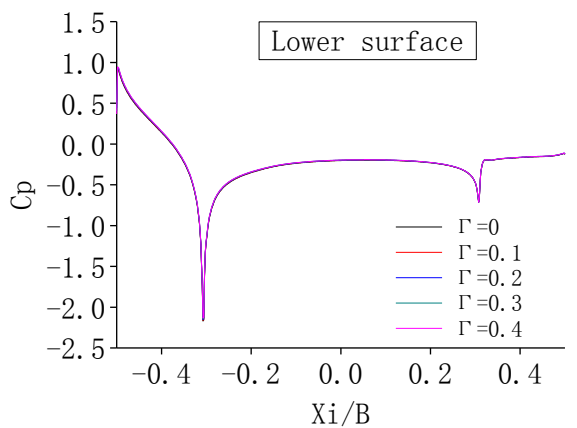

(a) Lower Surface

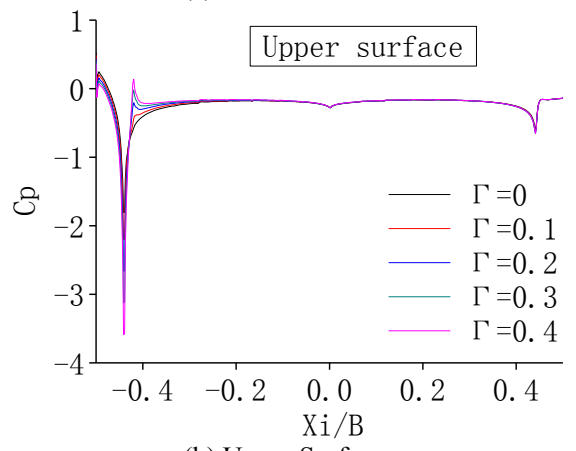

(b) Upper Surface

Fig.7 Mean Pressure Coefficient $C_{p}$ When AOA $\alpha=0^{\circ}$ for Case I

The drag force of the box girder is reduced by applying air suction as shown in Fig.8. The drag force is remarkably influenced by the air suction on the upper surface. For different AOA $\alpha$, the influence degree is not the same. When $\alpha=-6^{\circ}$, the drag-reducing is quite limited. As $\alpha$ increases, the drag rapidly decreases. When $\alpha=+6^{\circ}$, the drag force reduces to nearly a third of the no air suction case $(\Gamma=0)$.

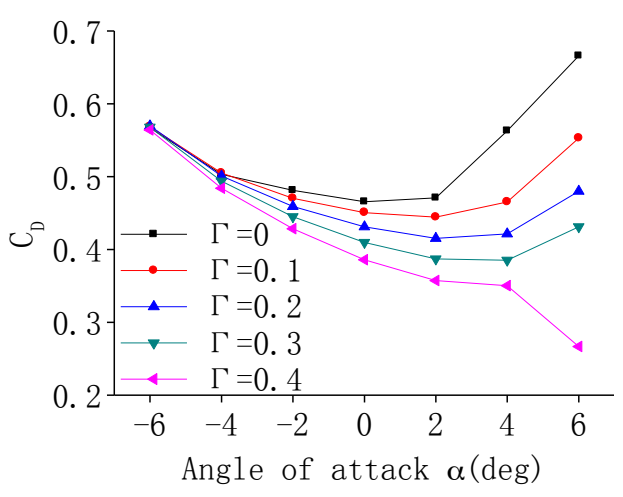

Fig.8 Drag Force Coefficient $C_{D}$ for Case I 
Tang Ke, Shi Cong, Zhou ZhiYong, Yang HanXiang and Sungur P.T

/Journal of Engineering Science and Technology Review 7 (2) (2014) 190 - 196

The difference of drag-reducing between positive and negative AOA is explained by pressure distribution in Fig.9. The suction method reduces the drag force by controlling the flow separated from girder section. When AOA is positive, the master separation occurs at the upper surface. So the upper surface air suction works validly. Once AOA is negative, the master separation occurs at the lower surface. The upper surface suction cannot directly influence the separation. As a result, the drag force shows no differences.

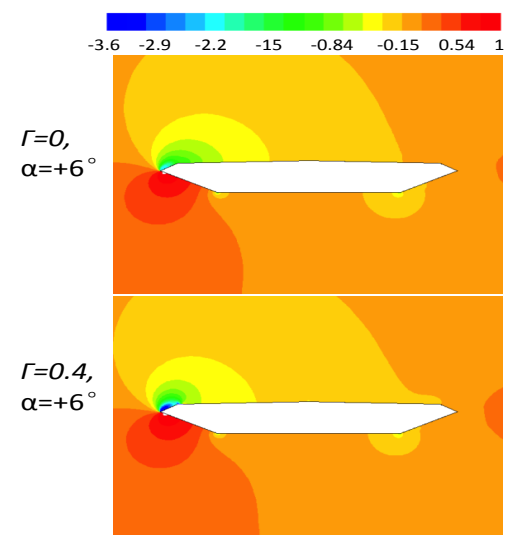

Fig.9 Contours of Mean Pressure Coefficient $C_{P}$ for Case I

As shown in Fig.10 and Fig.11, the mean lift force and moment seem not sensitive to the air suction.

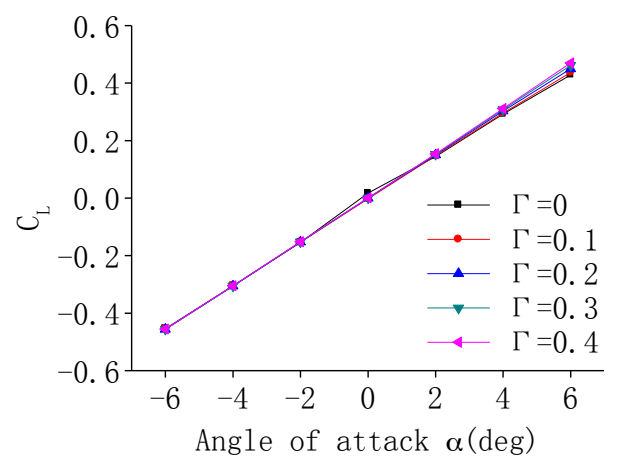

Fig.10 Lift Force Coefficient $C_{L}$ for Case I

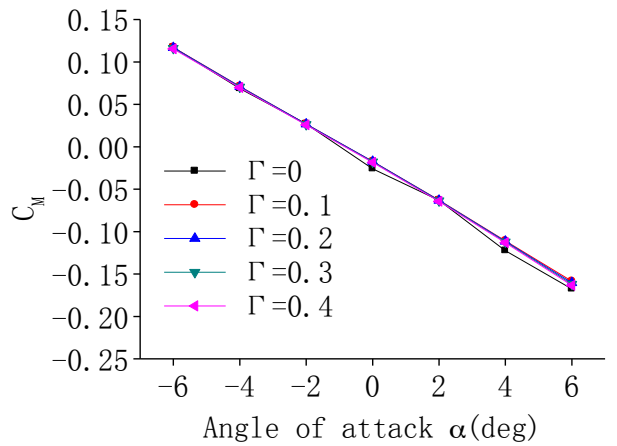

Fig.11 Moment Coefficient $C_{M}$ for Case I

The Strouhal(St) number of the flow is calculated from the fast Fourier transform (FFT) analysis of the time history of the vertical velocity at the recording point $\mathrm{x} / \mathrm{B}=2$ located downstream of the girder section. Since the Strouhal number is a global parameter which represents the nondimensional vortex shedding frequency, the location of recording point would have no influence.
Fig.12 exhibits the evolution of the St number with various suction ratios when AOA $\alpha=0^{\circ}$. When there is no air suction, the St value is found to be 0.163 . For the case of suction on upper surface, the St number is found to decrease from $\mathrm{St}=0.163$ to $\mathrm{St}=0.135$ approximately linearly when the suction ratio $\Gamma$ increases from 0 to 0.4 . At the higher suction ratio, the air suction leads to lower dynamical gradient in flow field. The lower gradients allow for slower dynamics, thus, lower vortex shedding frequency.

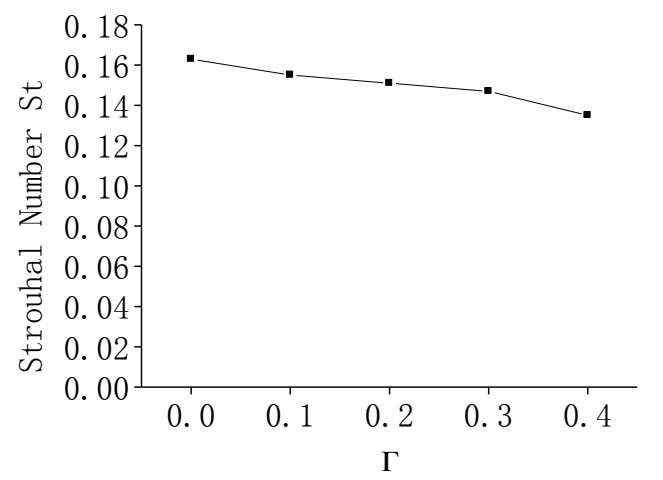

Fig.12 Variation of the Strouhal Number St with the Suction Ration

\subsection{Suction on Lower Surface (Case II)}

In this case, air suction between $\Gamma=0$ and $\Gamma=0.4$ is employed on the lower surface of girder. When AOA $\alpha=0^{\circ}$, the pressure coefficient distribution around the girder is depicted in Fig.13. Applying suction through the lower surface also provides approximately similar pressure distribution on the girder upper surface (Fig.13b). The flow above girder cannot be influenced by a lower surface suction. For the lower surface of girder (Fig.13a), the flow separation is somehow controlled by the suction. When the suction effect is stronger, for instance $\Gamma=0.4$, the magnitude of negative pressure on lower surface is less.

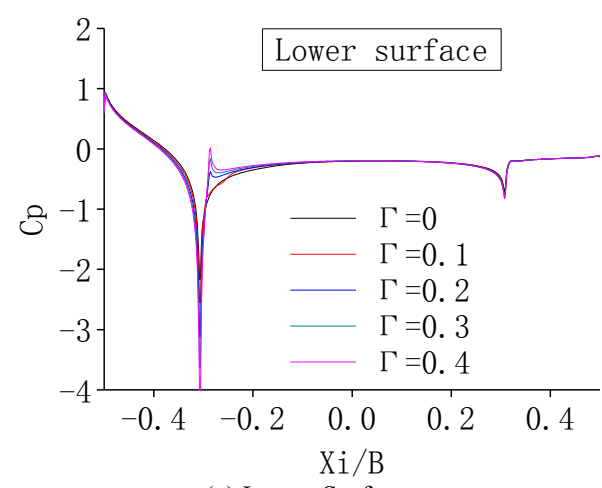

(a) Lower Surface

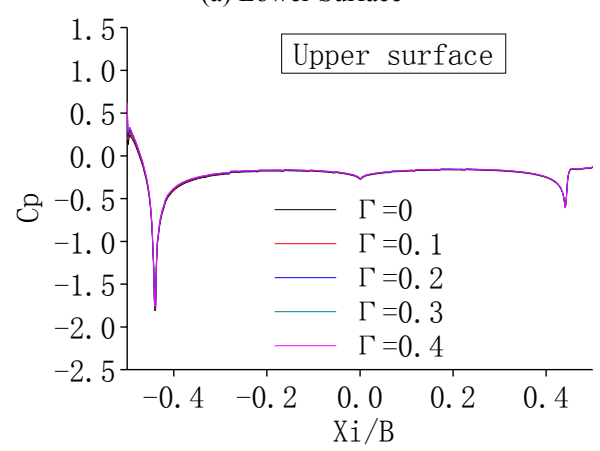

(b) Upper Surface

Fig.13 Mean Pressure Coefficient $C_{p}$ When the Angle of Attack $\alpha=0^{\circ}$ for Case II 
Tang Ke, Shi Cong, Zhou ZhiYong, Yang HanXiang and Sungur P.T

/Journal of Engineering Science and Technology Review 7 (2) (2014) 190 - 196

The drag force of the box girder is reduced by applying air suction as shown in Fig.14. The drag force is also influenced by the air suction on the upper surface. For different AOA $\alpha$, the influence is not the same. When $\alpha=+6$ ${ }^{\circ}$, the drag-reducing is just a little. As $\alpha$ decreases, the drag increases notably. When $\alpha=-6^{\circ}$, the drag force reduces to nearly two-thirds of the no air suction case.

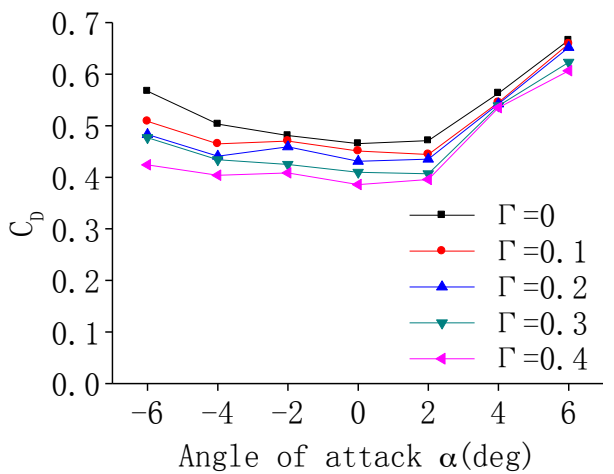

Fig.14 Drag Force Coefficient $C_{D}$ for Case II

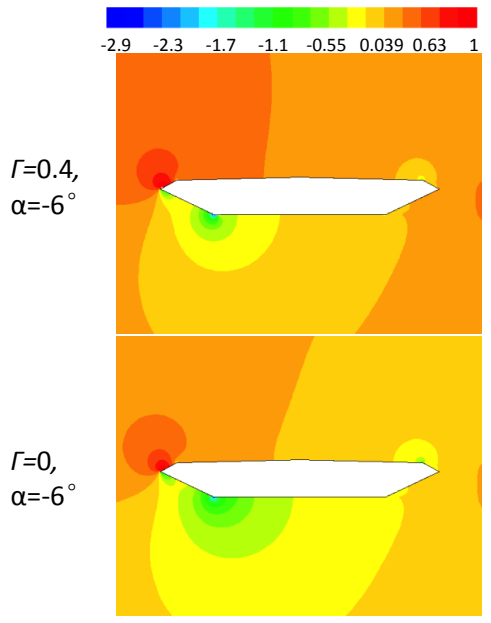

Fig.15 Contours of Mean Pressure Coefficient $C_{D}$ for Case II

The difference of drag-reducing between positive and negative AOA is explaned by the pressure distribution in Fig.15. When AOA is negative, the master separation occurs at the lower surface. Thus the lower surface suction can just work validly. Once AOA is positive, the master separation occurs at the upper surface. The lower surface suction cannot directly influence the separation. As a result, the drag force would be no differences.

The mean lift force and moment seem not sensitive to the air suction, seen in Fig.16 and Fig.17.

Fig.18 exhibits the evolution of the Strouhal number with various suction ratios when AOA $\alpha=0^{\circ}$. For the case of suction on lower surface, the Strouhal number also decreases from $\mathrm{St}=0.163$ to $\mathrm{St}=0.119$ approximately linearly when the suction ratio $\Gamma$ increases from 0 to 0.4 . Compared with case, suction on lower surface seems have stronger control of the wake flow around the girder. The cause lies in the fact that the vortex shedding near the lower surface dominates the whole wake flow of the girder due to the asymmetry of the cross section.

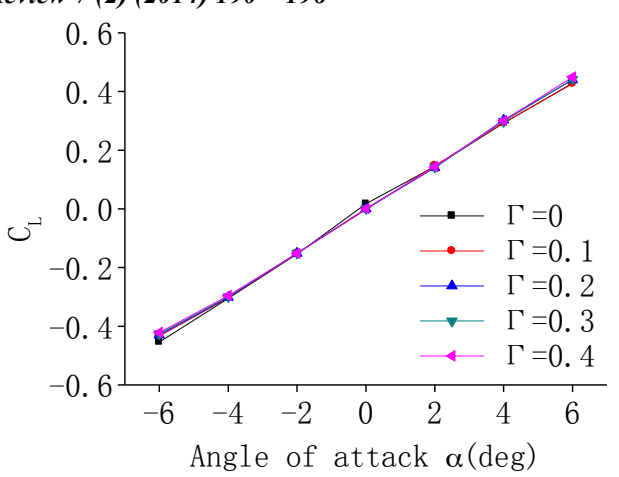

Fig.16 Lift Force Coefficient $C_{L}$ for Case II

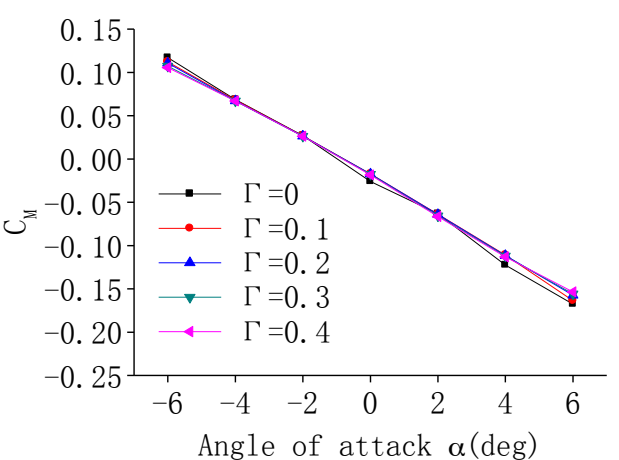

Fig.17 Moment Coefficient $C_{M}$ for Case II

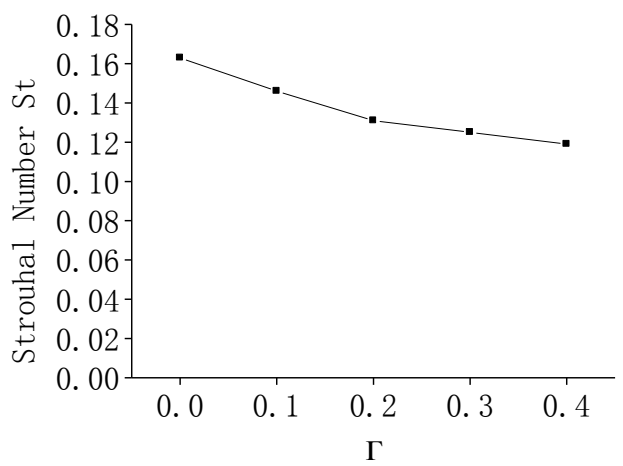

Fig.18 Variation of the Strouhal Number St with the Suction Ration for Case II

\section{Conclusions}

Flow around a streamlined box girder cross section with uniform air suction has been studied numerically. The main conclusions are as follows:

For conditions in which suction is only applied on the upper surface, the drag forces decreases when the suction ratio $\Gamma$ is increasing. When $\alpha=-6^{\circ}$, the drag-reducing is quite limited. As $\alpha$ increases, the drag rapidly decreases. When $\alpha=+6^{\circ}$, the drag force reduces to nearly a third of the no air suction case.

For conditions in which suction is only applied on the lower surface, the drag forces also decreases by increasing the suction ratio $\Gamma$. When $\alpha=+6^{\circ}$, the drag force reduces just a little. As $\alpha$ decreases, the drag increases notably. When $\alpha=-6^{\circ}$, the drag force reduces to nearly two-thirds of the no air suction case. 
Tang Ke, Shi Cong, Zhou ZhiYong, Yang HanXiang and Sungur P.T

/Journal of Engineering Science and Technology Review 7 (2) (2014) 190 - 196

The air suction method does not affect the aerodynamic lift force and moment of the steamlined box girder.

The Strouhal number is found to decrease approximately linearly with the suction ratio.
A optimal combination scheme that considering both suction position and suction ratio to reduce the aerodynamic drag force or to control the flow wake in engineering desgin would be more efficient.

\section{References}

1. Modi V J, Hill S S, Yokomizo T., "Drag reduction of trucks through boundary-layer control", Journal of Wind Engineering and Industrial Aerodynamics, 54, 1995, pp. 583-594.

2. Patil, .K.R.,Ng,T.T., "Control of separation using spanwise periodic porosity", AIAA Journal, 48(1) , 2010, pp. 174-187.

3. Ling Guoping, Fang Jianwen. "Numerical study on the flow around a circular cylinder with surface suction or blowing using vorticityvelocity method", Applied Mathematics and Mechanics, 23(9), 2002, pp.1089-1096.

4. Fransson, J. H. M., Konieczny, P., Alfredsson, P. H., "Flow around a porous cylinder subject to continuous suction or blowing", Journal of Fluids and structures, 19(8), 2004, pp. 1031-1048.

5. S.C. Luo, Roy X.Y. Tan, "Induced parallel vortex shedding from a circular cylinder at $\mathrm{Re}$ of $\mathrm{O}\left(10^{4}\right)$ by using the cylinder end suction technique", Experimental Thermal and Fluid Science, 33(8), 2009, pp. 1172-1179

6. Wen-Li Chen, Da-Bo Xin, Feng Xu, Hui Li, Jin-Ping Ou, Hui Hu, "Suppression of vortex-induced vibration of a circular cylinder using suction-based flow control", Journal of Fluids and Structures, 42, 2013, pp. 25-39

7. Tom Shtendel, Avi Seifert, "Three-dimensional aspects of cylinder drag reduction by suction and oscillatory blowing", International Journal of Heat and Fluid Flow, 45, 2014, pp. 109-127
8. Dong, S., Triantafyllou, G. S., Karniadakis, G. E, "Elimination of vortex streets in bluff-body flows", Physical Review Letters, 100(20), 2008, 204501-04.

9. Cuhadaroglu, B., Akansu, Y., Turhal, A, “An experimental study on the effects of uniform injection through one perforated surface of a square cylinder on some aerodynamic parameters", Experimental Thermal and Fluid science, 31(8), 2007, pp. 909915.

10. Çuhadaroğlu, B., Turan A., Osman, "Numerical Simulation of Turbulent Flow Around a Square Cylinder with Uniform Injection or Suction and Heat Transfer", Numerical Heat Transfer, Part A: Applications, 55(2), 2009, pp. 163 - 184.

11. Cuhadaroglu, B., "A numerical study on turbulent flow around a square cylinder with uniform injection or suction", International Journal of Numerical Methods for heat and Fluid Flow, 19(6), 2009, pp. 708-727.

12. Turhal, A., Çuhadaroğlu, B., "The effects of surface injection through a perforated square cylinder on some aerodynamic parameters", Experimental Thermal and Fluid science, 34(6), 2010, pp. 725-735

13. Menter, F. R., "Two-Equation Eddy-Viscosity Turbulence Models for Engineering Applications", AIAA Journal, 32(8), 1994, pp. 1598-1605. 